\title{
ESTRUCTURA DE CAPITAL Y RENTABILIDAD DEL SECTOR BANCARIO QUE OPERA EN EL PERÚ
}

\author{
CAPITAL STRUCTURE AND PROFITABILITY OF THE BANKING SECTOR THAT \\ OPERATES IN PERU
}

Valiente Pantaleón Ramírez Huerta Universidad Nacional Mayor de San Marcos Lima, Perú

ORCID: https://orcid.org/0000-0002-9297-056X Correo electrónico: vramiezh@yahoo.com

Edgar Vicente Armas Universidad Nacional Mayor de San Marcos Lima, Perú

ORCID: https://orcid.org/0000-0003-3112-5247 Correo electrónico: evicentea@unmsm.edu.pe

\section{RESUMEN}

Objetivo: Establecer la asociación del costo promedio ponderado de capital con el rendimiento sobre los activos, del costo alternativo de capital con el rendimiento sobre el patrimonio, y del costo promedio de deuda con el rendimiento sobre las ventas en los bancos que operan en el Perú. Método: Investigación de enfoque cuantitativo, no experimental-transversal y correlacional. La población estuvo formada por 18 bancos, no fue necesario calcular la muestra; se recolectaron los datos mediante el análisis documental de los estados financieros anuales de 2010 a 2018 de cada banco; las hipótesis se probaron mediante el índice de correlación. Resultados: Se determinaron el costo promedio ponderado de capital, de capital propio y de capital por deuda; también el índice de rendimiento sobre los activos sobre el patrimonio neto, sobre las ventas y el valor económico añadido. Conclusión: Existe una correlación inversa entre el costo de capital promedio ponderado y el rendimiento sobre los activos; también entre el costo neto de la deuda de escudo fiscal y el rendimiento sobre las ventas; sin embargo, existe correlación directa entre el costo alternativo de capital y el rendimiento sobre el capital propio.

Palabras clave: Banca; estructura de capital; costo de capital; tipos de rentabilidad; indicadores de creación de valor.
[Recibido: 21/11/2020 Aceptado: 03/05/2021 Publicado: 31/08/2021]

\begin{abstract}
Objective: Establish the association of the weighted average cost of capital with the return on assets, of the alternative cost of capital with the return on equity, and of the average cost of debt with the return on sales in banks operating in Peru. Method: The investigation had a quantitative approach, was non-experimental transversal, and correlational. The population consisted of 18 banks, and it was not necessary to calculate the sample. The data was collected through the documentary analysis of the annual financial statements from 2010 to 2018 of each bank; the hypotheses were tested using the correlation index. Results: The weighted average cost of capital, equity capital, and debt capital were determined; as well as the index of return on assets, on net worth, on sales, and on the added economic value. Conclusion: There is an inverse correlation between the weighted average cost of capital and the return on assets; also, between the cost of debt net of tax shield and the return on sales; however, there is a direct correlation between the alternative cost of capital and the return on equity.
\end{abstract}

Keywords: Banking; capital structure; cost of capital; types of profitability; value creation indicators. 


\section{INTRODUCCIÓN}

En el Perú, las empresas bancarias se constituyen como sociedades anónimas y se encuentran reguladas y supervisadas por una entidad gubernamental denominada Superintendencia de Banca, Seguros y Administradoras Privadas de Fondos de Pensiones (SBS). Los bancos fundamentalmente se dedican a la intermediación financiera entre los agentes económicos superavitarios de dinero y los deficitarios de ello.

En el país operan dieciocho empresas bancarias, diecisiete de ellas son de inversión privada y una del Estado peruano. Las tasas de interés exigidas por los bancos dependen del tipo de crédito que otorgan; así, las más altas son de créditos de consumo, seguida de créditos a pymes, y las más bajas corresponden a los créditos corporativos.

Acerca de la concentración bancaria, Martin (2009) afirma que es el "sistema bancario en el cual pocos bancos tienen la mayor participación, ya sea en depósitos, préstamos o en activos" (p. 165). En ese sentido, Uzcátegui, Camino y Moran (2017) sostienen que "existe evidencia de que dicho mercado en Ecuador posee una estructura oligopolística en donde existen 4 empresas (bancos) que controlan el 65\% del mercado desde el año 2012” (p. 12).

La actividad económica desarrollada por los bancos persigue la finalidad de generar rentabilidad gestionando eficientemente sus recursos controlados (activos); en ese sentido, el Banco Central de Reserva del Perú (2021) define la rentabilidad como "Capacidad de un activo para generar utilidad” (párr. 38). Interpretando a Ordoñez (2015), la rentabilidad económica es una medida de eficiencia para monitorear cómo es valorada una inversión en una actividad económica. Así, la inversión en la actividad bancaria será eficiente si logra generar beneficios para los inversionistas y otros acreedores. Adrianzen (2016) sobre la rentabilidad de bancos en el Perú concluye que "las variables representativas del entorno macroeconómico (la tasa de inflación, el crecimiento del PIB y el nivel de PIB por persona) resultaron determinantes recurrentemente significativos de la rentabilidad de los bancos comerciales que operaron en el Perú" (p. 183).

La generación de beneficios se mide con índices de rendimiento respecto a la inversión o las ventas. Forsyth (2012) afirma que "es preocupante para los inversionistas y directivos de las empresas la rentabilidad que se está obteniendo por el capital invertido, y con el propósito de estimarla se ha desarrollado una serie de indicadores" (p. 35). Los indicadores más usados son los siguientes:

- Rendimiento sobre los activos (ROA): Indicador para evaluar la óptima utilización de los activos de los bancos en la generación de ganancias. Para Mishkin
(2008) "un indicador básico de la rentabilidad del banco es el rendimiento sobre los activos (return on assets, ROA)” (p. 232).

$$
\mathrm{ROA}=\frac{\text { Utilidad Operativa } *(1-\text { tasa de impuestos })}{\text { Activo }} 100
$$

- $\quad$ Rendimiento sobre el capital (ROE): Es un indicador de eficiencia del banco en la generación de rendimiento sobre el capital propio a favor de los accionistas, según García (2015) "indica el porcentaje de utilidad neta que obtienen los propietarios sobre el capital invertido en la compañía” (p. 277).

$$
\mathrm{ROE}=\frac{\text { Utilidad neta }}{\text { Patrimonio }} 100
$$

- Rendimiento sobre las ventas (ROS): Revela el porcentaje de los ingresos por intermediación financiera y actividades conexas que se convierten en utilidad neta. Según García (2015) "el porcentaje de utilidad generada por cada unidad monetaria de ventas" ( $\mathrm{p}$. 175).

$$
\text { ROS }=\frac{\text { Utilidad neta }}{\text { Ingresos operativos }} 100
$$

- Valor Económico Añadido (EVA): Si resulta positivo, indica que se ha generado valor mediante el uso de los recursos controlados; caso contrario, valor destruido. Según Chu (2018), "el EVA corresponde a la diferencia entre la utilidad creada por la empresa y el costo alternativo de los recursos empleados para lograr esa utilidad" (p. 586), concordando con ello Stern y Shiely (2002) afirman que "es el beneficio que queda una vez deducido el coste del capital invertido para generar dicho beneficio" (p. 35).

$$
V E A=R O A-W A C C
$$

- Beneficio Económico para los Accionistas (BEA): Es el beneficio neto para los accionistas, este se encuentra sustrayendo del índice ROE el del COK, el cual es calculado mediante el modelo de fijación de precios de activos de capital (Capital Asset Pricing Model CAPM) que forma parte del WACC.

$$
B E A=R O E-C O K
$$

En síntesis, los indicadores de rentabilidad como ROA, ROE y ROS se encuentran relacionando rubros de los estados financieros. Sin embargo, el indicador EVA y BEA requieren incorporar el COK (costo de oportunidad del capital) en el primer caso, a través del WACC (Weighted Average Cost of Capital) y en el segundo directamente. 
Estos últimos indicadores miden la rentabilidad económica y financiera real de una compañía.

Por otra parte, la estructura de capital de una empresa bancaria se forma por el financiamiento por deuda y aporte de los accionistas. Para Forsyth (2012): “Cuando determinemos la estructura óptima de capital, debemos pensar en el capital estructural, permanente, que cubre las necesidades de mediano plazo en la empresa" (p. 171). Claro está, que cada fuente implica incurrir en costo de capital. Brealey, Myers y Allen (2010) afirman: "Definimos el costo de capital de la empresa como 'el rendimiento esperado de un portafolio que contiene todos los títulos existentes en la empresa'. Habitualmente, ese portafolio incluye deuda y capital propio" (p. 241). En el Perú, para el funcionamiento de las empresas bancarias a diciembre de 2018 se requería un capital social aportado en efectivo de S/ 27296676.00 (Superintendencia de Banca, Seguros y Administradora de Fondo de Pensiones, [SBS], 2018), condicionando por norma legal la estructura de capital.

Integrando los planteamientos precedentes afirmamos que la estructura de capital está conformada por una parte del financiamiento por pasivo corriente (corto plazo), el pasivo no corriente (deuda a mediano y largo periodo), además del patrimonio neto (capital contable). El financiamiento por deuda a largo plazo en los bancos se clasifica en créditos del público y préstamos de terceros; por su parte, el financiamiento por aportación está conformado por el capital y la retención de ganancias. Cada proveedor de fondos exige retribución; así el costo de las deudas se establece deduciendo a la tasa de interés la tasa del escudo fiscal, mientras que el costo del capital aportado se establece mediante la tasa de COK.

Sobre el costo de capital, Brealey et al. (2010) afirman que "se calcula como una mezcla del costo de la deuda (la tasa de interés) y el costo de capital propio (la tasa de rendimiento esperada exigida por los inversionistas en el capital ordinario)" (p. 241) y según Chu (2018) "el costo de capital promedio ponderado de muchas empresas es el promedio de los costos financieros de los préstamos a largo plazo más el costo de oportunidad del capital contable de la compañía” (p. 427). Así, es consenso en el campo de las finanzas en el uso de la siguiente fórmula:

$$
W A C C=\frac{E}{E+D} K e+\frac{D}{E+D} K d(1-T)
$$

En el que:

Ke: Tasa del COK
E/(E+D): Proporción del capital propio en el capital estructural

E/(E+D): Proporción de la deuda en el capital estructural

Kd: Tasa de costo del capital por deuda.

T: Tasa del impuesto a las ganancias más ponderación de participación de utilidades.

Para hallar el costo del capital propio, se usa generalmente el modelo CAPM que para Chu (2018) "es una herramienta financiera muy utilizada no por su exactitud, sino por las mejores prácticas en finanzas” (p. 410).

Con base en las citas precedentes se puede afirmar que la estructura de capital de una entidad bancaria se forma sumando el capital ajeno (pasivos permanentes) más el capital propio (patrimonio neto). El costo del capital (o gasto financiero) es la recompensa pactada por el uso el dinero con los acreedores e inversionistas (accionistas). Así, en el caso de los acreedores, es la tasa de interés menos el escudo fiscal, mientras que en el de los inversionistas, es la tasa de COK (costo alternativo); finalmente, el WACC se calcula sumando el producto de cada tasa con el porcentaje de cada tipo de capital en la estructura.

Según Modigliani y Miller (1963), la generación de beneficios en una compañía no está relacionada con la estructura de capital; sin embargo, existen imperfecciones sistemáticas en el mercado financiero como el impuesto a las ganancias que rompe dicha independencia. Así se infiere, que dicho impuesto incide en el costo de capital por deuda, pero no en el del capital propio. De ello se plantea la existencia de una estructura óptima de capital que debe lograrse en un periodo.

Sobre la velocidad de ajuste de la estructura de capital de empresas peruana, Cornejo (2014) sostiene que "se estima una velocidad de ajuste del nivel de endeudamiento del 33\%, lo cual implica que el ajuste completo al nivel de endeudamiento deseado es de alrededor de 3 años" (p. i). Por otra parte, sobre estructura de capital de empresas de Ecuador, Sinche (2016) concluye que "los ajustes medios alcanzan aproximadamente el 25,67\% y 47,74\% anual en el corto y largo plazo" (p. 41). Dichos estudios analizan periodos necesarios para alcanzar la estructura óptima de capital. Sobre la estructura de capital y el rol de la rentabilidad, Almahadin (2019) concluye que "el impacto de la estructura de capital parece ser de naturaleza complicada y difícil de examinar, sin controlar la interacción de la rentabilidad como uno de los principales determinantes" ( $p$. 379). En cuanto a la dinámica de estructura de capital y rentabilidad en el sistema bancario vietnamita, Pham et al. (2019) concluyen que la rentabilidad responde negativamente al choque de apalancamiento, lo que implica el 
uso ineficiente de este para elevar la utilidad neta durante el período de estudio; es decir, "el costo por intereses y los gastos por riesgo de incumplimiento superan el beneficio obtenido" (p. 2486).

De lo expuesto surge la interrogante: ¿De qué manera los costos de la estructura de capital están asociadas con los rendimientos generados en las empresas bancarias que operan en el Perú, en el periodo 2010-2018? Por lo tanto, se buscó establecer la asociación entre el costo promedio ponderado de capital y el rendimiento sobre los activos; entre el costo de oportunidad de capital y el rendimiento sobre el patrimonio; $y$ entre el costo promedio ponderado de deuda y el rendimiento sobre las ventas. De este modo, aportar evidencia empírica sobre las variables que permita el análisis y la discusión sobre políticas de Estado en este sector, es importante en la vida económica del país.

\section{MATERIAL Y MÉTODOS}

Fue una investigación de enfoque cuantitativo, no experimental, retrospectiva. Se aplicó el método deductivo. La población de estudio estuvo constituida por 18 bancos que se desenvolvieron en el Perú entre el año 2010 al 2018; no se requirió muestra, los elementos de análisis fueron las compañías bancarias. Se recurrió al análisis documental para la recolección de datos, se usó como instrumento fichas de resumen y la fuente de datos fueron los estados financieros anuales de cada banco, extraídos de la base de datos "Información financiera" (Superintendencia del Mercado de Valores [SMV], 2019). El procesamiento de datos y el análisis de la información se hicieron mediante el software MS-Excel y para probar la hipótesis se halló el índice de correlación entre las variables cruzadas en las hipótesis.

\section{RESULTADOS}

La participación de cada una de las compañías que operaron como parte del sector bancario peruano, mediante la proporción porcentual promedio anual de nueve años del activo, pasivo e ingreso, se detalla a continuación en la Tabla 1.

Entre el año 2010 al 2018 la inversión promedio en activos efectuados por todas las empresas bancarias que operan en el Perú fue: el Banco de Crédito invirtió el 32,70\%; seguido por el BBVA con 19,5\%; en tercer lugar, Scotiabank con $14,2 \%$; en cuarto lugar, Interbank con 10,5\%; y en quinto lugar, el Banco de la Nación con 7,9\%.

En la estructura financiera (pasivo y capital propio) del conjunto de empresas bancarias que operan en el Perú

Tabla 1

Participación en el mercado bancario peruano, periodo 2010-2018

\begin{tabular}{|c|c|c|c|c|c|c|}
\hline & Banco & Activo & Pasivo & Patrimonio & Estructura K & Ingreso \\
\hline 1 & BBVA & $19,52 \%$ & $22,92 \%$ & $17,14 \%$ & $22,9 \%$ & $17,75 \%$ \\
\hline 2 & Cencosud & $0,21 \%$ & $0,17 \%$ & $0,51 \%$ & $0,2 \%$ & $0,61 \%$ \\
\hline 3 & Citibank & $1,90 \%$ & $1,75 \%$ & $2,57 \%$ & $1,7 \%$ & $1,78 \%$ \\
\hline 4 & Comercio & $0,53 \%$ & $0,50 \%$ & $0,60 \%$ & $0,5 \%$ & $0,73 \%$ \\
\hline 5 & Crédito del Perú & $32,66 \%$ & $31,28 \%$ & $32,01 \%$ & $31,3 \%$ & $30,64 \%$ \\
\hline 6 & Pichincha & $2,18 \%$ & $2,13 \%$ & $1,73 \%$ & $2,1 \%$ & $2,48 \%$ \\
\hline 7 & Interamericano & $2,91 \%$ & $3,28 \%$ & $2,12 \%$ & $3,3 \%$ & $2,50 \%$ \\
\hline 8 & Interbank & $10,46 \%$ & $10,06 \%$ & $9,92 \%$ & $10,1 \%$ & $12,29 \%$ \\
\hline 9 & MiBanco & $2,57 \%$ & $2,41 \%$ & $3,05 \%$ & $2,4 \%$ & $4,80 \%$ \\
\hline 10 & Scotiabank & $14,21 \%$ & $13,16 \%$ & $17,96 \%$ & $13,2 \%$ & $11,95 \%$ \\
\hline 11 & GNB & $1,38 \%$ & $1,31 \%$ & $1,47 \%$ & $1,3 \%$ & $1,18 \%$ \\
\hline 12 & Falabella & $1,13 \%$ & $1,01 \%$ & $1,77 \%$ & $1,0 \%$ & $2,89 \%$ \\
\hline 13 & Santander & $1,23 \%$ & $1,17 \%$ & $1,24 \%$ & $1,2 \%$ & $0,75 \%$ \\
\hline 14 & Ripley & $0,54 \%$ & $0,46 \%$ & $1,02 \%$ & $0,5 \%$ & $1,63 \%$ \\
\hline 15 & Azteca & $0,23 \%$ & $0,21 \%$ & $0,32 \%$ & $0,2 \%$ & $0,95 \%$ \\
\hline 16 & Deutsche Bank & $0,25 \%$ & $0,20 \%$ & $0,55 \%$ & $0,2 \%$ & $0,26 \%$ \\
\hline 17 & Nación & $7,95 \%$ & $7,85 \%$ & $5,63 \%$ & $7,9 \%$ & $6,74 \%$ \\
\hline \multirow[t]{2}{*}{18} & ICBC Perú Bank & $0,15 \%$ & $0,12 \%$ & $0,39 \%$ & $0,1 \%$ & $0,07 \%$ \\
\hline & Total sector & $100 \%$ & $100 \%$ & $100 \%$ & $100 \%$ & $100 \%$ \\
\hline
\end{tabular}

Fuente: Elaboración propia con cálculo en el promedio aritmético de datos de 9 años (2010- 2018) de SMV (2019) 
encontramos que, a través del elemento pasivo el Banco de Crédito captó el 31,28\%; BBVA el 22,92\%; Scotiabank el 13,16\%; Interbank el 10,06\%; y en quinto lugar, el Banco de la Nación captó el 7,85\%. En cuanto al elemento capital propio, los accionistas del Banco de Crédito aportaron el 32\%; los de Scotiabank el 18\%; los de BBVA el 17,1\%; los de Interbank el 9,9\%; y los del Banco de la Nación el 5,6\%.

Respecto a la estructura de capital del sector, el Banco de Crédito aportó el 31,3\%; seguido por el BBVA con 22,9\%; Scotiabank, en tercer lugar, con 13,2\%; en cuarto lugar, Interbank con 10,1\%; y en el quinto lugar, el Banco de la Nación con $7,9 \%$.

En cuanto a los ingresos totales del sector, el Banco de Crédito participó del 30,6\% del mercado; seguido por BBVA con $17,8 \%$; luego Interbank con $12,3 \%$; y, finalmente, el Scotiabank con el 12\%; en conjunto participan del $73 \%$ del mercado. El Banco de la Nación, una entidad del Estado, participó del 6,7\% de los ingresos del mercado.

Los resultados antes descritos, en cuanto a la estructura económica, estructura financiera, de capital y los ingresos, indican fehacientemente que el sector bancario peruano es un oligopolio liderado por el Banco de Crédito, secundado por BBVA, Scotiabank e Interbank, todas ellas inversiones privadas. El Banco de la Nación, incluso que atiende a todo el sector gubernamental, aparece en el quinto lugar, pero muy relegado.

El costo anual promedio ponderado del capital estructural por cada empresa bancaria y del sector bancario se detalla en la Tabla 2.

El costo promedio neto anual de capital por deuda de escudo fiscal de los bancos que operaban en el Perú fue de $1,73 \%$ anual. Para los bancos privados que lideran el sector: Banco de Crédito 1,70\%, BBVA 1,53\%, Interbank 2,03\%, Scotiabank 1,46\%. Para el Banco de la Nación $0,4 \%$; se calcula relacionando gastos por intereses con los pasivos una vez deducidas las obligaciones a la vista.

Para calcular el COK se recurrió al CAPM, cuya fórmula usada fue:

$\mathrm{COK}=$ tasa libre de riesgo $+\left(\beta\right.$ local ${ }^{*} \beta$ apalancado ${ }^{*}$ prima de mercado USA) + EMBI

Donde:

- $\quad$ Tasa libre de riesgo $=3,26 \%$ (media aritmética de los rendimientos diarios de 2010 al 2018 de bonos a $30 \mathrm{Yr}$ del tesoro USA)

Tabla 2

Estructura y costo de capital promedio anual por banco del 2010 al 2018

\begin{tabular}{|c|c|c|c|c|c|}
\hline Banco & $\%$ de deuda & $\%$ de aporte & Costo deuda & COK & WACC \\
\hline BBVA & $90,76 \%$ & $9,24 \%$ & $1,53 \%$ & $18,34 \%$ & $3,08 \%$ \\
\hline Cencosud & $75,07 \%$ & $24,93 \%$ & $3,13 \%$ & $10,72 \%$ & $5,02 \%$ \\
\hline Citibank & $65,54 \%$ & $34,46 \%$ & $2,10 \%$ & $9,48 \%$ & $4,64 \%$ \\
\hline Comercio & $86,16 \%$ & $13,84 \%$ & $3,49 \%$ & $14,32 \%$ & $4,99 \%$ \\
\hline Crédito & $86,62 \%$ & $13,38 \%$ & $1,70 \%$ & $14,59 \%$ & $3,43 \%$ \\
\hline Pichincha & $91,34 \%$ & $8,66 \%$ & $2,48 \%$ & $19,14 \%$ & $3,92 \%$ \\
\hline BIF & $92,40 \%$ & $7,60 \%$ & $1,68 \%$ & $20,95 \%$ & $3,15 \%$ \\
\hline Interbank & $88,09 \%$ & $11,91 \%$ & $2,03 \%$ & $15,63 \%$ & $3,65 \%$ \\
\hline MiBanco & $87,65 \%$ & $12,35 \%$ & $2,86 \%$ & $15,29 \%$ & $4,39 \%$ \\
\hline Scotiabank & $83,29 \%$ & $16,71 \%$ & $1,46 \%$ & $12,92 \%$ & $3,38 \%$ \\
\hline GNB & $87,97 \%$ & $12,03 \%$ & $2,23 \%$ & $15,53 \%$ & $3,83 \%$ \\
\hline Falabella & $83,82 \%$ & $16,18 \%$ & $4,07 \%$ & $13,14 \%$ & $5,54 \%$ \\
\hline Santander & $88,68 \%$ & $11,32 \%$ & $1,50 \%$ & $16,11 \%$ & $3,16 \%$ \\
\hline Ripley & $80,26 \%$ & $19,74 \%$ & $5,03 \%$ & $11,90 \%$ & $6,38 \%$ \\
\hline Azteca & $85,25 \%$ & $14,75 \%$ & $3,64 \%$ & $13,82 \%$ & $5,14 \%$ \\
\hline Deutsche & $77,27 \%$ & $22,73 \%$ & $1,30 \%$ & $11,15 \%$ & $3,54 \%$ \\
\hline Nación & $85,34 \%$ & $14,66 \%$ & $0,40 \%$ & $13,86 \%$ & $2,37 \%$ \\
\hline ICBC Bank & $69,99 \%$ & $30,01 \%$ & $0,94 \%$ & $9,96 \%$ & $3,65 \%$ \\
\hline Promedio & $87,27 \%$ & $12,73 \%$ & $1,73 \%$ & $15,02 \%$ & $3,42 \%$ \\
\hline
\end{tabular}

Fuente: Elaboración propia con cálculo en el promedio aritmético de datos de 9 años (2010-2018) de SMV (2019) 
- $\quad$ Beta para entidades financieras en USA $=28 \%(\mathrm{Da}-$ modaran)

- $\quad$ Tasa de escudo fiscal $=32,965 \%$ (impuesto $29,5 \%$ y participaciones 5\%)

- $\quad$ Beta local $=41,01 \%$ (calculado con el rendimiento de la BVL y MSCI)

- $\quad \beta$ apalancado $=\beta$ activos $*\left(1+\left(\left(D / E^{*}(1-\right.\right.\right.$ tasa de escudo fiscal))

- Deuda / Equity según los estados financieros de cada banco

- Prima de mercado = promedio aritmético rendimiento (S\&P) 5,96\%

- $\quad \mathrm{EMBI}=5.68 \%-3.26 \%=2,42 \%$ (promedio del rendimiento diario entre el 2010 al 2018 de bonos del estado peruano en soles a 10 años menos la tasa libe de riesgo. Calculado con datos publicados por la autoridad monetaria (Banco Central de Reserva del Perú [BCRP], 2019).
El promedio del costo anual de oportunidad del capital aportado por los accionistas de los bancos que operan en el Perú fue de 15,02\% anual. Para los bancos privados que lideran el sector: Banco de Crédito 14,59\%, BBVA 18,34\%, Interbank 15,63\%, Scotiabank 12,92\%; y para el Banco de la Nación $13,86 \%$.

El WACC de las empresas representa el costo de la estructura de capital (recursos financieros permanentes). Para el sector bancario peruano entre el año 2010 al 2018, se ha estimado en 3,42\% anual. En cuanto a los bancos privados que lideran el sector se ha estimado: para el Banco de Crédito en 3,43\%; para el BBVA, en 3,08\%; para el Interbank, en 3,65\%; para Scotiabank, en 3,38\%. En cuanto a la entidad del Estado, el Banco de la Nación tiene un WACC de $2,37 \%$ anual, se encuentra por debajo del promedio del sector.

Los niveles de rendimiento promedio anual generado por cada uno de los bancos en 9 años (2010-2018) y del sector bancario en su conjunto se revelan en la Tabla 3.

El margen de utilidad operativa respecto a los ingresos totales es un beneficio que la intermediación financiera y los servicios financieros pueden generar; se calcula

Tabla 3

Indicadores de rendimiento por banco del 2010 al 2018

\begin{tabular}{|c|c|c|c|c|c|c|}
\hline Banco & Activo & $\begin{array}{l}\text { Resultado } \\
\text { operación }\end{array}$ & $\begin{array}{r}\text { Utilidad } \\
\text { neta }\end{array}$ & ROA & ROE & ROS \\
\hline BBVA & 62479479 & 1764147 & 1289166 & $1,93 \%$ & $22,73 \%$ & $28,41 \%$ \\
\hline Cencosud & 673445 & -16214 & 2405 & $-1,61 \%$ & $1,43 \%$ & $1,54 \%$ \\
\hline Citibank & 6091228 & 129589 & 117302 & $3,53 \%$ & $13,82 \%$ & $25,81 \%$ \\
\hline Comercio & 1702717 & 28658 & 22036 & $1,34 \%$ & $11,13 \%$ & $11,75 \%$ \\
\hline Crédito & 104523381 & 2909155 & 2193460 & $2,46 \%$ & $20,71 \%$ & $28,01 \%$ \\
\hline Pichincha & 6971876 & 52803 & 45264 & $0,53 \%$ & $7,89 \%$ & $7,14 \%$ \\
\hline BIF & 9303359 & 130523 & 90794 & $0,95 \%$ & $12,96 \%$ & $14,19 \%$ \\
\hline Interbank & 33489405 & 962965 & 738019 & $2,34 \%$ & $22,48 \%$ & $23,49 \%$ \\
\hline MiBanco & 8233009 & 227688 & 169117 & $1,87 \%$ & $16,75 \%$ & $13,79 \%$ \\
\hline Scotiabank & 45469503 & 1274952 & 977181 & $2,40 \%$ & $16,44 \%$ & $31,99 \%$ \\
\hline GNB & 4411986 & 5958 & 12307 & $0,10 \%$ & $2,54 \%$ & $4,09 \%$ \\
\hline Falabella & 3615675 & 118156 & 97318 & $2,19 \%$ & $16,65 \%$ & $13,20 \%$ \\
\hline Santander & 3923231 & 65716 & 46353 & $1,22 \%$ & $11,33 \%$ & $24,04 \%$ \\
\hline Ripley & 1723938 & 48792 & 61053 & $1,91 \%$ & $18,02 \%$ & $14,69 \%$ \\
\hline Azteca & 726348 & 15281 & 11199 & $1,42 \%$ & $10,50 \%$ & $4,60 \%$ \\
\hline Deutsche & 795389 & 32893 & 24577 & $2,77 \%$ & $13,59 \%$ & $37,53 \%$ \\
\hline Nación & 25440128 & 661070 & 561491 & $3,49 \%$ & $30,14 \%$ & $32,60 \%$ \\
\hline ICBC Bank & 495197 & -5607 & -5531 & $-0,87 \%$ & $-4,29 \%$ & $-32,20 \%$ \\
\hline Promedio & 17781628 & 467029 & 358528 & $2,17 \%$ & $19,51 \%$ & $25,25 \%$ \\
\hline
\end{tabular}

Fuente: Elaboración propia con cálculo en el promedio aritmético de datos de 9 años (2010- 2018) de SMV (2019) 
sustrayendo de la utilidad bruta, los gastos de administración y de ventas. Así, el margen de utilidad operativa promedio del sector bancario entre el año 2010 al 2018 fue de $32,9 \%$. Entre los bancos privados que lideran el sector, el Banco de Crédito obtuvo un margen de utilidad operativo de 37,2\%; el BBVA de 38,9\%; Interbank de 30,6\%; Scotiabank de 41,7\%. Para el Banco de la Nación (estatal) fue de $38,4 \%$.

El rendimiento sobre el activo total (ROA) promedio de los últimos nueve años del sector en el Perú fue 2,17\%. El activo total comprende todos los recursos controlados y destinados a la ejecución de la actividad económica de intermediación financiera y los servicios financieros. El indicador muestra que, por cada cien soles invertidos en el banco, se generó $2,17 \%$ de utilidad promedio anual.

Mientras que, el rendimiento respecto al capital contable (ROE) promedio de los últimos nueve años del sector en análisis fue del 19,51\%. El capital contable (patrimonio neto) lo conforman los aportes de capital y resultados retenidos; es decir, es la inversión efectuada por los accionistas de cada empresa bancaria, por lo tanto, el indicador muestra que por cada cien soles invertidos, los accionistas obtuvieron 19,51 soles de utilidad anual en promedio.
El rendimiento sobre las ventas (ROS) de los últimos nueve años del sector en estudio fue de $25,25 \%$, indicando el tanto por ciento de los ingresos convertidos en utilidad neta atribuible a los accionistas. Así, en el presente estudio, por cada cien soles de ingreso que generan los bancos, veinticinco soles se convierten en utilidad neta.

Entre los bancos privados que lideran el sector, el Banco de Crédito obtuvo un ROA de 2,46\%, un ROE de 20,71\%, y un ROS de $28,01 \%$. Por su parte el BBVA obtuvo un ROA de $1,93 \%$, un ROE de $22,73 \%$, y un ROS de $28,41 \%$. Por otra parte, Interbank obtuvo un ROA de $2,34 \%$, un ROE de 22,48\%, y un ROS de 23,49\%. Finalmente, Scotiabank obtuvo un ROA de 2,40\%, un ROE de 16,44\%, y un ROS de 31,99\%. El Banco de la Nación, que representa al Estado, obtuvo un ROA de 3,49\%, un ROE de $30,14 \%$, y un ROS de $32,60 \%$.

El VEA y el BEA promedio anual generado por cada uno de los bancos y del sector bancario en su conjunto, se detalla en la Tabla 4.

El EVA nos indica el valor agregado generado o el valor destruido por un sector o una entidad con los recursos que tuvo bajo su control. En el periodo de análisis, se encontró que el sector bancario peruano muestra un índice

Tabla 4

Valor económico agregado y beneficio económico por banco (2010 - 2018)

\begin{tabular}{lrrrrrr}
\hline \multicolumn{1}{c}{ Banco } & ROA & WACC & EVA & ROE & CAPM & BEA \\
\hline BBVA & $1,93 \%$ & $3,08 \%$ & $-1.15 \%$ & $22,3 \%$ & $18,34 \%$ & $4,39 \%$ \\
Cencosud & $-1,61 \%$ & $5,02 \%$ & $-6.63 \%$ & $1,43 \%$ & $10,72 \%$ & $-9,53 \%$ \\
Citibank & $3,53 \%$ & $4,64 \%$ & $-1.12 \%$ & $13,82 \%$ & $9,48 \%$ & $4,34 \%$ \\
Comercio & $1,34 \%$ & $4,99 \%$ & $-3.65 \%$ & $11,13 \%$ & $14,32 \%$ & $-3,19 \%$ \\
Crédito & $2,46 \%$ & $3,43 \%$ & $-0.96 \%$ & $20,71 \%$ & $14,59 \%$ & $6,12 \%$ \\
Pichincha & $0,53 \%$ & $3,92 \%$ & $-3.39 \%$ & $7,89 \%$ & $19,14 \%$ & $-11,25 \%$ \\
BIF & $0,95 \%$ & $3,15 \%$ & $-2.20 \%$ & $12,96 \%$ & $20,95 \%$ & $-7,99 \%$ \\
Interbank & $2,34 \%$ & $3,65 \%$ & $-1.31 \%$ & $22,48 \%$ & $15,63 \%$ & $6,86 \%$ \\
MiBanco & $1,87 \%$ & $4,39 \%$ & $-2.53 \%$ & $16,75 \%$ & $15,29 \%$ & $1,46 \%$ \\
Scotiabank & $2,40 \%$ & $3,38 \%$ & $-0.97 \%$ & $16,44 \%$ & $12,92 \%$ & $3,52 \%$ \\
GNB & $0,10 \%$ & $3,83 \%$ & $-3.73 \%$ & $2,54 \%$ & $15,53 \%$ & $-12,99 \%$ \\
Falabella & $2,19 \%$ & $5,54 \%$ & $-3.35 \%$ & $16,65 \%$ & $13,14 \%$ & $3,50 \%$ \\
Santander & $1,22 \%$ & $3,16 \%$ & $-1.94 \%$ & $11,33 \%$ & $16,11 \%$ & $-4,78 \%$ \\
Ripley & $1,91 \%$ & $6,38 \%$ & $-4.48 \%$ & $18,02 \%$ & $11,90 \%$ & $6,13 \%$ \\
Azteca & $1,42 \%$ & $5,14 \%$ & $-3.73 \%$ & $10,50 \%$ & $13,82 \%$ & $-3,32 \%$ \\
Deutsche & $2,77 \%$ & $3,54 \%$ & $-0.76 \%$ & $13,59 \%$ & $11,15 \%$ & $2,44 \%$ \\
Nación & $3,49 \%$ & $2,37 \%$ & $1.11 \%$ & $30,14 \%$ & $13,86 \%$ & $16,28 \%$ \\
ICBC Bank & $-0,87 \%$ & $3,65 \%$ & $-4.52 \%$ & $-4,29 \%$ & $9,96 \%$ & $-14,25 \%$ \\
\hline Promedio & $2,17 \%$ & $3,42 \%$ & $-1.26 \%$ & $19,51 \%$ & $15,02 \%$ & $4,49 \%$ \\
\hline
\end{tabular}

Fuente: Elaboración propia con cálculo en el promedio aritmético de datos de 9 años (2010-2018) de SMV (2019) 
de EVA de -1,26\%, lo cual indica que se ha destruido valor; es decir, que por cada 100 soles de activos en los bancos se destruye el valor por 1,26 soles en promedio. El EVA para los bancos privados que lideran el sector fue: Banco de Crédito -0,96\%, el BBVA -1,15\%, Interbank - $1.31 \%$, y Scotiabank $-0,97 \%$; todos son negativos lo que indica que el rendimiento obtenido sobre los activos no cubre el costo del capital ajeno y propio invertido. En cambio, el EVA generado por el Banco de la Nación fue 1,11\%, lo cual indica que ha generado un valor añadido de 1,11\%.

El beneficio económico para los accionistas (BEA) muestra la creación de valor a favor de los accionistas o la destrucción de valor. En el periodo de análisis, encontramos que el sector bancario peruano muestra un índice de BEA que fue de $4,49 \%$, lo cual indica que se ha creado valor para los accionistas. Se puede interpretar que, por cada 100 soles invertidos por los accionistas de los bancos, se agregó valor por 4,49 soles por encima del COK.

En cuanto al índice de BEA de los bancos privados que lideran el sector, se tiene al Banco de Crédito con 6,12\%; BBVA con 4,39\%; Interbank con $6,86 \%$; y Scotiabank con $3,32 \%$. Todos son positivos, lo cual indica que el rendimiento obtenido sobre el capital propio (perteneciente a las accionistas) excede su costo alternativo. El índice de BEA del Banco de la Nación fue 16,28\%, ello indica que el rendimiento obtenido por capital aportado por los accionistas excede el COK.

\section{Prueba de hipótesis}

Se ha utilizado el índice "r" calculado con la siguiente fórmula:

Correlación $(X, Y)=\frac{n \cdot \sum x_{i} y_{i}-\left(\sum x_{i}\right)\left(\sum y_{i}\right)}{\sqrt{\left.\left[n \cdot \sum x_{i}^{2}-\left(\sum x_{i}\right)^{2}\right)\right]} \cdot \sqrt{\left.\left[n \cdot \sum y_{i}^{2}-\left(\sum y_{i}\right)^{2}\right)\right]}}$

Ha1: El WACC está asociada con el ROA en las empresas del sector bancario peruano 2010-2018:

El índice de correlación de dichas variables fue $-0,158$; por encontrarse cerca a cero indica que las variaciones del WACC están asociadas inversamente con el del ROA, es decir, son dependientes, por ello se acepta la Ha1.

Ha2: El COK está asociada con el ROE en las empresas del sector bancario peruano 2010-2018.

El índice de correlación de las variables mencionadas fue 0,228 , que mostró asociación directa; es decir, si el COK aumenta el ROE también lo hace. Se acepta la Ha2.
Ha3: El costo de capital promedio ponderado de deuda está asociada con el ROS en las empresas del sector bancario peruano 2010-2018.

El índice de correlación de las variables cruzadas fue $-0,214$, lo que significa relación inversa, permitiendo sostener que cuando el costo de la deuda aumenta el ROS disminuye. Se acepta la Ha3.

\section{DISCUSIÓN}

La concentración en una actividad económica se da cuando pocas empresas tienen la mayor proporción de la oferta total. En el periodo comprendido del 2010 al 2018 operaron 18 empresas bancarias en el Perú, pero el 76,9\% de la estructura económica (activos), el 74\% estructura financiera (pasivo y patrimonio neto), el 77,5\% a la estructura de capital, el 72,7\% de los ingresos generados en el sector lo concentran 4 bancos privados (BCP, BBVA, Scotiabank e Interbank). Por lo tanto, el mercado bancario peruano es oligopólico. Este hallazgo es similar a lo encontrado por Uzcátegui et al. (2017) en el mercado bancario ecuatoriano entre el 2000 y el 2015, en donde se observa que 4 empresas bancarias controlan el $65 \%$ de dicho mercado.

Se ha encontrado que la estructura de capital de los 18 bancos se encuentra apalancada, pues el financiamiento por deuda promedio en el sector representa el $84 \%$. El valor económico agregado generado por el sector fue $-1,26 \%$ (2,17\% - 3,42\%; ROA - WACC) y el beneficio económico generado para los accionistas fue 4,49\% (19,51\%-15,02\%; ROE - COK). Lo cual permite afirmar que los bancos estructuraron su capital permanente para maximizar la generación de beneficios a favor de sus accionistas; sin embargo, destruyen el valor para los ahorristas, quienes financian el capital estructural por deuda. Sin duda es necesario evaluar la legislación bancaria en un contexto de concentración de mercado para reformularla y así fortalecer el sistema financiero a favor de la sociedad peruana en su conjunto.

Existe correlación directa o inversa de las variables estudiadas, los índices de correlación fueron: 1) WACC vs $\mathrm{ROA}=-0.158 ; 2) \mathrm{COK}$ vs $\mathrm{ROE}=0.228,3)$ Costo de deuda vs ROS $=-0.214$, indican correlación débil (están muy distante a 10 -1) esto concuerda con el hallazgo de Pham et al. (2019) quienes sostienen que la rentabilidad responde negativamente al choque de apalancamiento; evidenciando la existencia de una relación inversa entre rentabilidad y estructura de capital. La débil correlación de las variables concuerda con Adrianzen (2016), quien afirma que la tasa de inflación y la variación del PBI total y del per cápita fueron determinantes de la rentabilidad en los bancos que operaron en el Perú, y con Almahadin (2019), quien explica que el impacto de la estructura de 
capital parece ser de naturaleza complicada y difícil de examinar, sin controlar la interacción de la rentabilidad como uno de sus determinantes.

Finalmente, se formulan las siguientes interrogantes para futuras investigaciones: ¿Cuál es la celeridad de ajuste de la estructura el capital de bancos en el Perú? ¿Cómo gestionar el banco para la generación de valor agregado a favor de los accionistas, ahorristas y otros acreedores?

\section{REFERENCIAS}

Adrianzen, C. (2016). La rentabilidad de los bancos comerciales y el ambiente macroeconómico: el caso peruano en el periodo 1982-2014 (Tesis doctoral). Universitat Politécnica de Catalunya, Barcelona.

Almahadin, H. A., \& Oroud, Y. (2019). Capital structure-firm value nexus: The moderating role of profitability. Revista Finanzas y Política Económica, 11(2), 371-382. http://dx.doi.org/10.14718/revfinanzpolitecon.2019.11.2.9

Banco Central de Reserva del Perú. (2021). Glosario de términos económicos. Recuperado de: https://www. bcrp.gob.pe/publicaciones/glosario/r.html

Banco Central de Reserva del Perú. (2019). Rendimiento del bono del gobierno peruano a 10 años (en s/). Recuperado de: https://estadisticas.bcrp.gob.pe/estadisticas/series/diarias/resultados/PD31893DD/html

Brealey, R., Myers S., \& Allen F. (2010). Principios de finanzas corporativas (9a ed.). México D.F.: McGraw-Hill.

Cornejo, R. (2014). Estructura de capital en mercados emergentes. Velocidad de ajuste de la estructura de capital en las empresas peruanas cotizadas en bolsa (Tesis de PhD.). Universidad Ramon Llull, Barcelona.

Chu, J. (2018). Fundamentos de Finanzas un enfoque peruano (9a ed.). Lima: Advisory.

Forsyth, A. (2012). Finanzas empresariales: rentabilidad y valor. (2a ed. y 2. ${ }^{a}$ reimpresión). Lima: Autor.

García, V. (2015). Analisis financieros un enfoque integral. México D.F: Grupo editorial Patria.
Martin, M. (2009). Transparencia y concentración bancaria en el Perú. México D.F: Pearson Educación.

Mishkin, F. (2008). Moneda, banca y mercados financieros. (8a ed.). México D.F: Pearson Educación.

Modigliani, F., \& Miller, M. (1963). Corporate Income Taxes and the Cost of Capital: A Correction. The American Economic Review, 53(3), 433-443. Recuperado de: http://www.jstor.org/stable/1809167

Ordoñez, M. (2015). Indagación teórica respecto al concepto de eficacia organizacional. Tendencias \& Retos, 20(2), 101-117.

Pham, M. P., Hoang, C. V., Tran, K., Vu, H. V., Thi Thuy, L., \& Nguyen, B. C. (2019). Dynamics of capital structure, risk-taking behavior, and profitability across the vietnamese banking system: Evidence from a panel vector autoregression. Journal of Advanced Research in Law and Economics, 10(8), 2474-2489. https://doi. org/10.14505/jarle.v10.8(46).25

Sinche, R. (2016). Análisis Dinámico de la Estructura de capital de las empresas de Ecuador: Modelo de ajuste parcial (Tesis de maestría). Universidad de Chile, Santiago de Chile.

Stern, J., \& Shiely, J. (2002). El reto del EVA. Cómo implantar y Gestionar el cambio del valor añadido en una organización. Barcelona: Ediciones Gestión 2000.

Superintendencia de Banca, Seguros y Administradoras Privadas de Fondos de Pensiones. (2018). Actualización del capital social mínimo correspondiente al trimestre octubre - diciembre de 2018 (Circular No G-1992018). Lima: Autor.

Superintendencia del Mercado de Valores. (2019). Información financiera. Recuperado de: http://www. smv.gob.pe/Frm_InformacionFinanciera.aspx?da$\mathrm{ta}=202 \mathrm{D} 3 \mathrm{E} 90 \mathrm{E} 2 \mathrm{C} 3948 \mathrm{EC} 3 \mathrm{D} 46 \mathrm{ADAF} 23 \mathrm{CBD} 3 \mathrm{FC}$ 34CEAAE9C

Uzcátegui, C., Camino, S., \& Moran, J. (2017). Estructura de mercado del sistema bancario ecuatoriano: concentración y poder de mercado. Cumbres, 4(1), 49- 62. 